\title{
Energy Conservation and the Manley-Rowe Relations in Surface Nonlinear-Optical Spectroscopy
}

\author{
A. V. Petukhov, ${ }^{1}{ }^{*}$ Vera L. Brudny,${ }^{2}$ W. Luis Mochán, ${ }^{3}$ Jesús A. Maytorena, ${ }^{4}$ Bernardo S. Mendoza, ${ }^{5}$ and Th. Rasing ${ }^{1}$ \\ ${ }^{1}$ Research Institute for Materials, University of Nijmegen, 6525 ED Nijmegen, The Netherlands \\ ${ }^{2}$ Departamento de Física “J. J. Giambiaggi," Facultad de Ciencias Exactas y Naturales, Universidad de Buenos Aires, \\ Ciudad Universitaria, Pabellón I, 1428 Buenos Aires, Argentina \\ ${ }^{3}$ Laboratorio de Cuernavaca, Instituto de Física, Universidad Nacional Autónoma de México, Apartado Postal 48-3, \\ 62251 Cuernavaca, Morelos, México \\ ${ }^{4}$ Facultad de Ciencias, Universidad Autónoma del Estado de Morelos, Avenida Universidad 1001, \\ 62210 Cuernavaca, Morelos, México \\ ${ }^{5}$ Centro de Investigaciones en Optica, León, Guanajuato, México
}

(Received 27 January 1998)

\begin{abstract}
We consider the energy conservation problem for nonlinear-optical three-wave mixing processes at the surface and in the bulk of semi-infinite centrosymmetric lossless media. Because of the intrinsic nonlocality of these processes, the corresponding Manley-Rowe relations differ from those of noncentrosymmetric media. The ensuing restrictions link the surface and bulk nonlinear susceptibility components and may be used to test theories of nonlinear response. Our analysis permits a clear separation of the intrinsic surface contribution that contains the information about the surface structure. [S0031-9007(98)06647-2]
\end{abstract}

PACS numbers: 42.65. $-\mathrm{k}, 41.20 . \mathrm{Bt}, 78.66 .-\mathrm{w}$

The energy conservation law is one of the basic principles in physics. In nonlinear optics the energy exchange between light waves is controlled by the Manley-Rowe (MR) relations, which require an equal number of photons of each mode participating in the nonlinear interaction [1]. Because of their general character, the energy conservation law and the MR relations do not depend on the detailed structure of a particular system, and their consequences are therefore very powerful. For example, they result in the well-known overall permutation symmetry of the nonlinear-optical susceptibility tensor $\overleftrightarrow{\chi}$ of a nonlinear nonabsorbing bulk medium [1,2], which, however, appears not to be obeyed at the surface. Because the surface nonlinear optics is now an important experimental tool in surface science [3], this apparent breaking of the energy conservation poses a very relevant fundamental problem with important practical applications.

In this Letter we reexamine the problem of the energy exchange between three interacting light waves at the surface and in the bulk of semi-infinite centrosymmetric nonlinear media. It is shown that the paradoxical result mentioned previously can be understood with the aid of an additional contribution to the energy flow that goes beyond the Poynting vector. The form of this energy flow is derived in general, and its physical origin is illustrated for two simple model systems. We find that additional restrictions on the surface and bulk nonlinear polarizabilities are required to ensure energy conservation. A part of the surface response is found to be determined by the bulk parameters alone. Our analysis allows clear separation of the "intrinsic" surface-originated part of the nonlinear-optical response that is sensitive to the details of the surface structure.
The second-order nonlinear optical techniques of threewave mixing $\left(\omega_{1}+\omega_{2}=\omega_{3}\right)$, second harmonic generation $\left(\omega_{1}=\omega_{2}\right)$ and sum frequency generation $\left(\omega_{1} \neq \omega_{2}\right)$ (SHG and SFG, respectively), combine the usual advantages of an optical probe with a strongly enhanced sensitivity to the interface electronic and magnetic structure of centrosymmetric media [2,3]. The latter stems from the fact that the second-order nonlinear interactions are dipole forbidden in the presence of inversion symmetry so that the bulk-originated part of the response is largely suppressed. At the surface, however, the inversion symmetry is broken, leading to a large surface-induced nonlinear susceptibility $\left(\chi^{S}\right)^{i j k}\left(-\omega_{3} ; \omega_{1}, \omega_{2}\right) \equiv\left(\chi^{S}\right) \frac{j}{3} 12$. An enormous progress has been achieved in experimental applications of the surface nonlinear-optical spectroscopy to study different systems [4]. On the other hand, although a number of theoretical studies of surface SHG [5-8] and a few extensions of the theory to surface SFG spectroscopy [9-11] have been reported, there are still various unsolved problems in understanding the fundamental physics of the surface nonlinear interactions. For threewave interactions in centrosymmetric bulk media the energy relations were first considered by Pershan [12], who constructed the free energy for transparent centrosymmetric systems with quadrupole-allowed nonlinearity. Agranovich and Darmanyan [13] incorporated the surface contribution into consideration, but under the assumption of a frequency independent response.

For illustration, we consider the well-known example of three-wave mixing in a noncentrosymmetric bulk medium illuminated by three electromagnetic waves $\tilde{\mathbf{E}}_{I}(\mathbf{r}, t)=$ $\mathbf{E}_{I}(\mathbf{r}) e^{-i \omega_{I} t}+\mathbf{E}_{\bar{I}}(\mathbf{r}) e^{i \omega_{I} t}$ with $I=1,2,3$. We shall indicate the frequency dependence of any physical quantity 
by an index $I, J$, or $K$, which may take any value $1,2,3$, subject to the convention that the frequencies $\omega_{I}, \omega_{J}, \omega_{K}$ might be either positive or negative and must fulfill the condition $\omega_{I}+\omega_{J}+\omega_{K}=0$ (e.g., negative $\omega_{I}$ may indicate the generation of a mode at $\omega_{I}$ while a mode with positive $\omega_{I}$ is absorbed). The energy taken from the $I$ th electromagnetic mode is given by the time-averaged work per unit volume [1]

$$
\mathcal{P}_{I}=\left\langle\tilde{\mathbf{j}}_{I}(r, t) \cdot \tilde{\mathbf{E}}_{I}(r, t)\right\rangle=-2 \omega_{I} \operatorname{Im}\left(\mathbf{P}_{\bar{I}} \cdot \mathbf{E}_{I}\right),
$$

where $\tilde{\mathbf{j}}_{I}(\mathbf{r}, t) \equiv \partial \tilde{\mathbf{P}}_{I}(\mathbf{r}, t) / \partial t$ is the current density induced at frequency $\omega_{I}$ and $\langle\ldots\rangle$ denotes the time average. The MR relations for a dissipationless process mixing the three waves $E_{I}^{i}$ read [1]

$$
\mathcal{P}_{I} / \omega_{I}=\mathcal{P}_{J} / \omega_{J}=\mathcal{P}_{K} / \omega_{K},
$$

which means that an equal number of photons of each mode participate in the nonlinear interaction. Since $\omega_{I}+\omega_{J}+\omega_{K}=0$, Eq. (2) guarantees the local energy conservation $\mathcal{P}_{I}+\mathcal{P}_{J}+\mathcal{P}_{K}=0$. The nonlinear polarization can be written in terms of the nonlinear susceptibility as $P_{\bar{I}}^{i}=\chi_{I J K}^{i j k} E_{J}^{j} E_{K}^{k}$. The MR relations then yield the overall permutation symmetry for the dipole nonlinear susceptibility

$$
\chi_{I J K}^{i j k}=\chi_{J K I}^{j k i}=\chi_{K I J}^{k i j} .
$$

However, examination of the existing theoretical results $[10,11]$ shows that the surface nonlinear susceptibility $\chi^{S}$ does not obey the overall permutation symmetry (3). For instance, for simple model flat surfaces like jellium [5] or continuous dipolium [7] surfaces, a tangential field does not induce a response in the normal direction; hence $\left(\chi^{S}\right)_{I J K}^{\perp\|\| \|}=0 . \quad$ On the other hand, $\left(\chi^{S}\right)_{I J K}^{\|\| \|} \neq 0$, since a normal external field $E_{K}^{\perp}$ induces a screening charge which is moved by the tangential field $E_{J}^{\|}$and thus gives rise to a tangential current at frequency $\omega_{K}+\omega_{J}$ $[10,11]$. As schematically illustrated in Fig. 1, the energy conservation law and the MR relations then seem to break down in surface nonlinear interactions.

In centrosymmetric bulk media the main contribution to the nonlinear polarization $\mathbf{P}_{I}$ can be described within the quadrupole approximation [3]

$$
P \frac{i}{I}=\chi_{I J K}^{i j l k} E_{J}^{j} \partial^{l} E_{K}^{k}+\chi_{I K J}^{i k l j} E_{K}^{k} \partial^{l} E_{J}^{j} .
$$

A direct substitution of this polarization into (1) shows that the work $\mathcal{P}_{I}$ done by the $I$ th wave depends on the spatial derivatives of $\mathbf{E}_{J}$ and $\mathbf{E}_{K}$ but not of $\mathbf{E}_{I}$. On the other hand, $\mathcal{P}_{J}$ does depend on the spatial variation of $\mathbf{E}_{I}$ but not of $\mathbf{E}_{J}$, etc. Therefore, the Manley-Rowe relations in the form of Eq. (2) cannot be fulfilled for an arbitrary spatial dependence of the fields. More strikingly, the sum of the works $\mathcal{P}_{I}+\mathcal{P}_{J}+\mathcal{P}_{K}$ done by the three electromagnetic modes can be nonvanishing, i.e., electromagnetic energy can be locally gained or lost in centrosymmetric dissipationless nonlinear media. Where does this energy come from or go to?

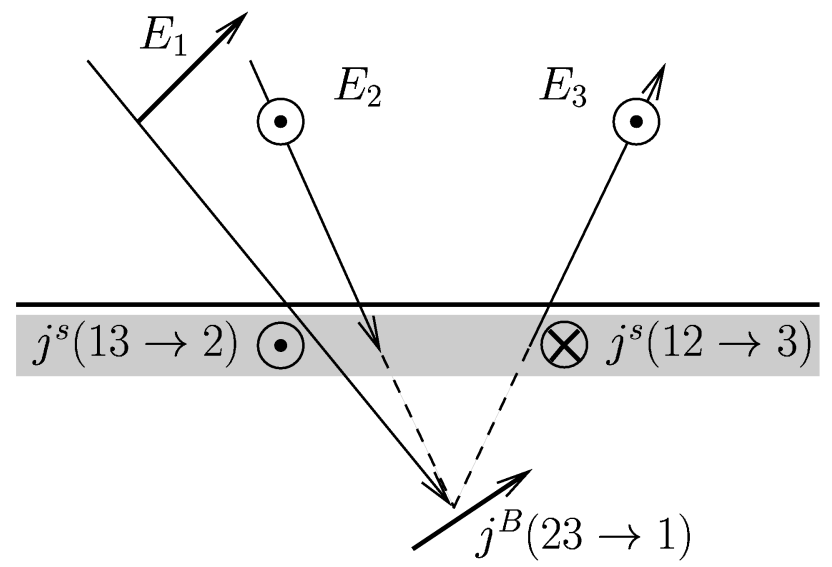

FIG. 1. Apparent violation of the energy conservation law at the selvedge (shaded area) of a flat isotropic surface. The fields (heavy arrows) $E_{1}$ and $E_{2}$ of the incident $p$ - and $s$-polarized waves, of frequencies $\omega_{1}$ and $\omega_{2}$, respectively, induce a surface current $j^{s}(12 \rightarrow 3)$ at $\omega_{3}=\omega_{1}+\omega_{2}$ which yields the energy source for the $s$-polarized outgoing field $E_{3}$. Similarly, $E_{1}$ and $E_{3}$ induce a current $j^{s}(13 \rightarrow 2)$, which yields the energy sink for the $\omega_{2}$ mode. However, there is no energy sink for the $\omega_{1}$ wave at the surface since $\left(\chi^{S}\right)_{12 \overline{3}}^{\perp\|\| \|}=0$. Instead, this energy is absorbed in the bulk. To restore the energy conservation law, additional contributions (dashed lines) to the energy flow have to be postulated, in addition to the usual electromagnetic flow (light arrows).

The solution to this problem starts from the realization that the bulk nonlinear polarization (4) essentially arises due to spatial nonlocality, i.e., the nonlinear polarization at $\mathbf{r}$ depends on the field at $\mathbf{r}^{\prime} \neq \mathbf{r}$. This nonlocal nonlinear interaction results in an additional contribution $\vec{S}_{I}$ to the energy flow that goes beyond the usual Poynting vector [13]. The time-averaged energy balance per unit volume related to the $I$ th electromagnetic mode should then be written as

$$
\mathcal{P}_{I}=W_{I}+\nabla \cdot \vec{S}_{I},
$$

which states that the energy taken from the electromagnetic field $\mathcal{P}_{I}$ is either frequency converted $\left(W_{I}\right)$ or transformed into a nonelectromagnetic energy flow $\vec{S}_{I}$. It is only $W_{I}$, and not $\mathcal{P}_{I}$, that should obey the Manley-Rowe relation

$$
W_{I} / \omega_{I}=W_{J} / \omega_{J}=W_{K} / \omega_{K} .
$$

In accordance with Eq. (4), $\vec{S}_{I}$ can be written in the form

$$
S_{I}^{l}=\omega_{I} F_{I J K}^{l i j k} E_{I}^{i} E_{J}^{j} E_{K}^{k},
$$

where $F_{I J K}^{l i j k}$ is a material tensor to be determined. By definition $F_{I J K}^{l i j k}=F_{I K J}^{l i k j}$ is symmetric with respect to the simultaneous interchange of the index pairs $J j$ and $K k$. To ensure fulfillment of the Manley-Rowe relation (6) for any combination of field values and their spatial derivatives within the nonabsorbing centrosymmetric bulk, the flow tensor must obey 


$$
F_{I J K}^{l i j k}-F_{J K I}^{l j k i}=i \chi_{I K J}^{i k l j}
$$

This leads to two sets of restrictions on the susceptibility: an antisymmetry relation

$$
\chi_{I J K}^{i j l k}=-\chi_{K J I}^{k j l i}
$$

and the Bianchi identity

$$
\chi_{I J K}^{i j l k}+\chi_{J K I}^{j k l i}+\chi_{K I J}^{k i l j}=0 .
$$

We note that Eq. (8) determines the nonelectromagnetic energy flow tensor

$$
F_{I J K}^{l i j k}=\frac{i}{3}\left(\chi_{I J K}^{i j l k}+\chi_{I K J}^{i k l j}\right)+G_{I J K}^{l i j k}
$$

up to an arbitrary tensor $G_{I J K}^{l i j k}$ which is completely symmetric in the three pairs of indices $I i, J j, K k$ and therefore does not contribute to the total nonelectromagnetic energy transport $\vec{S}_{I}+\vec{S}_{J}+\vec{S}_{K}$.

The energy deposited by the $I$ th mode of the electromagnetic field within the surface region is obtained by integrating across the selvedge, $\mathcal{P}_{I}^{S}=$ $\left\langle\int \tilde{\mathbf{j}}_{I}(\mathbf{r}, t) \cdot \tilde{\mathbf{E}}_{I}(\mathbf{r}, t) d z\right\rangle$, where the $z$ axis is normal to the surface. Both $\tilde{\mathbf{j}}_{I}(\mathbf{r}, t)$ and $\tilde{E}_{I}^{z}$ change rapidly within the interface region, which is assumed to be much thinner than the wavelength so that retardation effects can be neglected. We then approximate $\tilde{E}_{I}^{i}$ in terms of the field on the vacuum side of the interface $\left(E_{I}^{i}\right)_{\text {out }}$ and the depolarization field [5-7] to rewrite

$$
\mathcal{P}_{I}^{S}=-2 \omega_{I} \operatorname{Im}\left[\left(P^{S}\right)_{I}^{i}\left(E_{I}^{i}\right)_{\text {out }}\right]
$$

where

$$
\left(P^{S}\right) \frac{i}{I}=\int P_{\bar{I}}^{i}(\mathbf{r}) d z=\left(\chi^{S}\right)_{I J K}^{i j k}\left(E_{J}^{j} E_{K}^{k}\right)_{\mathrm{out}}
$$

is the total dipole moment of the surface per unit area at frequency $-\omega_{I}$. Thus,

$$
\mathcal{P}_{\bar{I}}^{S}=-2 \omega_{I} \operatorname{Im}\left[\left(\chi^{S}\right)_{I J K}^{i j k}\left(E_{I}^{i} E_{J}^{j} E_{K}^{k}\right)_{\text {out }}\right] .
$$

This energy is either frequency converted $\left(W_{I}^{S}\right)$ or transported into (from) the bulk in a nonelectromagnetic form. The energy flux is given by Eq. (7) where the fields $\left(E_{I}^{i}\right)_{\text {in }}=\eta_{I}^{i i^{\prime}}\left(E_{I}^{i^{\prime}}\right)_{\text {out }}$ are evaluated inside the nonlinear medium and are related to the fields outside via a screening tensor $\eta_{I}^{i i^{\prime}}$ (for isotropic surfaces it is diagonal with $\eta_{I}^{\|\| \|}=1$ and $\eta_{I}^{\perp \perp}=1 / \epsilon_{I}$, where $\epsilon_{I}$ is the dielectric function). The Manley-Rowe relations (6) applied to $W_{I}^{S}$ lead then to a relationship between the surface and the bulk susceptibility tensors,

$$
\left(\chi^{S}\right)_{I J K}^{i j k}=S_{I J K}^{i j k}+B_{I J K}^{i j k},
$$

where

$$
B_{I J K}^{i j k}=\frac{1}{3}\left(\chi_{I J K}^{i^{\prime} j^{\prime} z k^{\prime}}+\chi_{I K J}^{i^{\prime} k^{\prime} z j^{\prime}}\right) \eta_{I}^{i^{\prime} i} \eta_{J}^{j^{\prime} j} \eta_{K}^{k^{\prime} k}
$$

is totally determined by the bulk nonlinear parameters and does not depend on any detail of the surface structure. On the other hand,

$$
S_{I J K}^{i j k}=\frac{1}{3}\left[\left(\chi^{S}\right)_{I J K}^{i j k}+\left(\chi^{S}\right)_{J K I}^{j k i}+\left(\chi^{S}\right)_{K I J}^{k i j}\right]
$$

can be assigned to the intrinsic surface response. Unlike the total surface response $\left(\chi^{S}\right)_{I J K}^{i j k}$, it obeys the same overall permutation symmetry (3) as a true dipolar quadratic susceptibility. Since it has less independent components than $\left(\chi^{S}\right)_{I J K}^{i j k}$, it is more useful for surface diagnostics. The presence of a surface-independent contribution to $\chi^{S}$ was noted before by Guyot-Sionnest and Shen [14] for the particular case of surface SHG. Their analysis was based on the splitting of the nonlinear bulk response $\mathbf{P}$ into a "pure-electric-dipole" and an electric quadrupole polarization similar to Eq. (18) below. In this work we derived the bulk-originating contribution to the surface $\chi^{S}$ in a model-independent way suitable for any nonabsorbing medium, such as, for instance, a gas of freely moving electrons where the introduction of the quadrupole polarization is ambiguous.

The results derived above lead to some unexpected connections. In the study of surface SHG it is customary to introduce two parameters, $a(\omega)$ and $b(\omega)$, that are directly related to the $\left(\chi^{S}\right)^{\perp \perp \perp}$ and $\left(\chi^{S}\right)^{\|\| \| \perp}$ component of the susceptibility tensor, respectively [15]. For SFG, this parametrization is generalized to quantities that depend on the three frequencies involved, namely, $a_{I J K}$ and $b_{I J K}$. According to the jellium model $[10,11]$ the parameter $b_{I J K}$ takes the value -1 , but this need not be true for other systems. However, a more general statement can be made based on the relations derived here. It follows [16] from Eqs. (15)-(17) that $b_{I J K}=-1$ if and only if $\left(\chi^{S}\right)_{I J K}^{\perp\|\|}=0$. Similarly, a general conclusion can be drawn about the parameter $a_{I J K}$ : Eqs. (15)-(17) allow us to establish that it should attain the high frequency limit $a_{I J K} \rightarrow-2$ in a model independent way [16].

In a uniform isotropic free-electron gas (jellium) [5] the kinetic energy of a moving electron $m v^{2} / 2$ is transported by its own motion, leading to the energy flux $\mathbf{v} n m v^{2} / 2$ [17], where $n$ denotes the electron density. Thus, in the presence of three electromagnetic waves the physical origin of the nonelectromagnetic energy flow $\vec{S}$ can be understood as a convective transport of the kinetic energy $\vec{S}_{I}=\mathbf{v}_{I}\left(n m \mathbf{v}_{J} \cdot \mathbf{v}_{K}\right)+$ c.c., where $\mathbf{v}_{I}$ denotes the contribution to the electron velocity which is linear in the field of $I$ th electromagnetic mode.

We also consider another model system, dipolium [6,7], which consists of a semi-infinite distribution of small polarizable entities, so that in contrast to jellium the electrons are strongly confined. The macroscopic polarization of such a medium can be written in terms of a multipolar expansion [12]

$\mathbf{P}_{\bar{I}}=n_{B} \mathbf{d}_{\bar{I}}-\left(\frac{1}{2} \nabla \cdot n_{B} \overleftrightarrow{q}_{\bar{I}}+\frac{i c}{\omega_{I}} \nabla \times n_{B} \mathbf{m}_{\bar{I}}\right)$

where $n_{B}$ is the atomic density and $\mathbf{d}, \overleftrightarrow{q}$, and $\mathbf{m}$ are the electric dipole, quadrupole, and magnetic dipole of a single entity. From Eqs. (9) and (10) we find that the nonelectromagnetic energy flow is due to the electricquadrupolar $n_{B} \overleftrightarrow{q} \bar{I}$ and the magnetic-dipolar $n_{B} \mathbf{m}_{\bar{I}}$ 
polarizations of the medium via $\vec{S}=-n_{B} \operatorname{Re}\left(2 c \mathbf{E}_{I} \times\right.$ $\left.\mathbf{m}_{\bar{I}}+\mathbf{E}_{I} \cdot \partial \overleftrightarrow{q}_{\bar{I}} / \partial t\right)[12,16]$. We also note that within the dipolium model the surface-independent contribution $B_{I J K}^{i j k}$ to $\left(\chi^{S}\right)_{I J K}^{i j k}$ can thus be understood as an effect of the electric-quadrupolar and magnetic-dipolar polarizations of the bulk, which contribute to the electric-dipolar polarization at the surface [14].

In conclusion, in the present work we obtained a general solution to the long-standing problem of energy exchange between electromagnetic waves in surface nonlinear spectroscopy. Since three-wave mixing in centrosymmetric media is an intrinsically nonlocal process, an additional contribution $\vec{S}_{I}$ to the energy flow beyond the usual Poynting vector is required to account for the correct local energy balance. New energy-conserving Manley-Rowe relations were then derived, which lead to connections between the surface and bulk nonlinear susceptibilities. As a result, the surface nonlinear tensor $\overleftrightarrow{\chi}_{I J K}^{S}$ can be separated into two parts: One of them obeys the overall permutation symmetry (3) of the ordinary dipolar quadratic susceptibility and can be assigned to the surface specific response. The remaining part does not obey the symmetry (3) and is completely determined by the bulk response; therefore it is not suitable for surface diagnostics. The physical origin of the nonelectromagnetic energy flux $\vec{S}_{I}$ was illustrated for simple model systems. Compliance with our fundamental energy-conserving relations constitutes a test for theories of surface nonlinear-optical spectroscopy. Based on these relations, we have also derived limiting values for nonlinear-optical parameters at an isotropic surface. Since our results reduce the number of independent susceptibility components required to describe three-wave mixing at surfaces, we believe they might also prove useful for experimental data analysis.

This present work was partially supported by HCM, the TMR network "NOMOKE" of the EU, Technologiestichting STW, CONACyT-México (26651-E), DGAPAUNAM IN107796, and UBACYT-Argentina.

*Email address: petukhov@sci.kun.nl

FAX: +31-24-3652190.

On leave from the Institute of Crystallography, 117333 Moscow, Russia.
[1] P. N. Butcher and D. Cotter, The Elements of Nonlinear Optics (Cambridge University Press, Cambridge, 1990).

[2] Y.R. Shen, The Principles of Nonlinear Optics (Wiley, New York, 1984).

[3] For recent reviews, see Y.R. Shen, Solid State Commun. 102, 221 (1997); Th. Rasing, J. Magn. Magn. Mater. 175, 35 (1997); J.E. McGilp, J. Phys. D 29, 1812 (1996); G. A. Reider and T.F. Heinz, in Photonic Probes of Surfaces, edited by P. Halevi (Elsevier, Amsterdam, 1995), p. 413.

[4] P. Guyot-Sionnest, Phys. Rev. Lett. 67, 2323 (1991); Q. Du et al., ibid. 70, 2313 (1993); W. Daum et al., ibid. 71, 1234 (1993); A. Peremans and A. Tadjeddine, ibid. 73, 3010 (1994); C. Meyer et al., ibid. 74, 3001 (1995); B. Koopmans et al., ibid. 74, 3692 (1995); J. R. Power et al., ibid. 75, 1138 (1995); M. Straub, R. Vollmer, and J. Kirschner, ibid. 77, 743 (1996); J. Bloch, J. G. Mihaychuk, and H. M. van Driel, ibid. 77, 920 (1996); X. Su et al., ibid. 77, 3858 (1996); A. Kirilyuk et al., ibid. 77, 4608 (1996); J. Hohlfeld et al., ibid. 78, 4861 (1997).

[5] M. Weber and A. Liebsch, Phys. Rev. B 35, 7411 (1987); A. Liebsch, Phys. Rev. Lett. 61, 1233 (1988); A. Liebsch and W.L. Schaich, Phys. Rev. B 40, 5401 (1989); J. A. Maytorena et al., ibid. 51, 2556 (1995).

[6] W. L. Schaich and B. S. Mendoza, Phys. Rev. B 45, 14279 (1992); B. S. Mendoza and W. L. Mochán, ibid. 55, 2489 (1997).

[7] B. S. Mendoza and W. L. Mochán, Phys. Rev. B 53, 4999 (1996).

[8] A. V. Petukhov and A. Liebsch, Surf. Sci. 334, 195 (1995); H. Ishida, A. V. Petukhov, and A. Liebsch, ibid. 340, 1 (1995).

[9] J. Y. Huang and Y.R. Shen, Phys. Rev. A 49, 3973 (1994).

[10] A. V. Petukhov, Phys. Rev. B 52, 16901 (1995).

[11] J. A. Maytorena, B. S. Mendoza, and W. L. Mochán, Phys. Rev. B 57, 2569 (1998); J. A. Maytorena, W. L. Mochán, and B. S. Mendoza, ibid. 57, 2580 (1998).

[12] P. S. Pershan, Phys. Rev. 130, 919 (1963).

[13] V. M. Agranovich and S. A. Darmanyan, Pis'ma Zh. Eksp. Teor. Fiz. 35, 68 (1982) [JETP Lett. 35, 80 (1982)].

[14] P. Guyot-Sionnest and Y. R. Shen, Phys. Rev. B 38, 7985 (1988).

[15] J. Rudnick and E. A. Stern, Phys. Rev. B 4, 4272 (1971); J. E. Sipe, et al., ibid. 21, 4389 (1980).

[16] V. L. Brudny et al. (to be published).

[17] L. D. Landau and E. M. Lifshitz, Fluid Mechanics, Course of Theoretical Physics Vol. 6 (Pergamon, Oxford, 1993) 2nd English ed., Chap. I, Sect. 6. 